Mechani cal approach to agi ng and wri nkl i ng of human faci al ski $n$ based on the mil ti stage buckl ing theory

\begin{tabular}{|l|l|}
\hline 著者 & $\begin{array}{l}\text { KUMAZURU Osamu, SAOTHONG Jar i yapor n, YOSH KAWA } \\
\text { Nobuhi r o }\end{array}$ \\
\hline $\begin{array}{l}\text { j our nal or } \\
\text { publ i cat i on t i t l e }\end{array}$ & Nedi cal Engi neer i ng \& Phy si cs \\
\hline vol ume & 30 \\
\hline page r ange & $516-522$ \\
\hline year & 2008 \\
\hline URL & ht t p: //hdl . handl e. net /10098/6929 \\
\hline
\end{tabular}




\title{
Mechanical approach to aging and wrinkling of human facial skin based on the multistage buckling theory
}

\author{
Osamu Kuwazuru $^{\mathrm{a}, *}$, Jariyaporn Saothong ${ }^{\mathrm{b}}$, Nobuhiro Yoshikawa ${ }^{\mathrm{a}}$ \\ ${ }^{a}$ Institute of Industrial Science, The University of Tokyo, 4-6-1 Komaba, Meguro-ku, Tokyo 153-8505 Japan \\ ${ }^{\mathrm{b}}$ Industrial Engineering Department, Ubon Rajathanee University, P.O. Box 3, Warinchumrab, Ubonratchathani 34190 Thailand
}

\begin{abstract}
A wrinkle formation mechanism with cutaneous aging is addressed through a mechanical calculation of linear buckling. Skin is divided into five mechanically distinct layers in this study. In general, the outer layer is stiffer than the inner layer, so buckling occurs in the outer layer against the uniform compression caused by muscle contraction. This buckling damages the skin and affects the formation of permanent wrinkles. We propose a multistage buckling theory for evaluation of the wrinkle property, namely, the specific wrinkle size and critical strain in three stages. The specific wrinkle size is derived as the wavelength of the minimum-buckling mode for infinite-length skin. A sensitivity analysis is carried out to investigate the effect of age-related changes of the mechanical parameters on the wrinkle property. We employ some aging hypotheses and prepare two sets of mechanical parameters, one for young and one for aged skin. The numerical results show that the buckling mode switch from Stage I to Stage II is the main reason why pronounced wrinkles suddenly appear in aged skin.
\end{abstract}

Keywords: Skin; Aging; Wrinkle; Linear Buckling; Timoshenko Beam; Two-Parameter Foundation

\section{Introduction}

Antiaging has been considered a keyword or a dream of longevity medicine in the 21st century [1-4]. The basic concept of antiaging is that the enhancement of physical and mental health increases the physical resistance to most diseases, and improves the longevity and quality of life. For mental health, one's personal appearance, such as skin condition and body shape, is often very important. Of course, serious cutaneous disorders such as nevus and melasma are of the utmost importance in dermatological therapy [5-10]. For cutaneous antiaging, the interest is focused on aesthetic or cosmetic problems such as wrinkles and laxity, that is, the problems of skin beauty.

Skin wrinkles sometimes cause psychological pain, so there are many dermatological or surgical therapies for anti-wrinkle [5-10]. Beautiful smooth skin gives a person a juvenescent appearance and may affect the person's lifestyle. Wrinkle reduction leads to youthful skin and also a youthful mind, especially in regard to facial skin. The most important anti-wrinkle therapy is the prevention of aging. For this purpose, we studied the

\footnotetext{
* Corresponding author. Tel.: +81-3-5452-6105; fax: +81-3-54526105.

E-mail address: kuwa@telu.iis.u-tokyo.ac.jp (O. Kuwazuru)
}

wrinkle formation mechanism as a process of aging.

Conventional approaches to cutaneous aging are based mainly on physiological or biochemical observations $[11,12]$. The goal of these approaches is to reveal the histological structure of skin and its alteration with aging. Another approach is based on physical measurement to assess the age-related change in the mechanical properties of human skin [13]. However, the mechanical properties obtained in this approach are strongly dependent on the testing system [14,15], and the layered heterogeneity of skin has not been considered. As an exception, Maeno et al. [16] showed the three-layered mechanical properties of skin by an in vitro experiment. Hendriks et al. [17-19] have also tried to identify the two-layered mechanical properties by an in vivo numerical-experimental method.

For studies on skin wrinkles, many researchers have examined the superficial geometry or histological structure of wrinkled skin [11]. For instance, Lagarde et al. [20] proposed a numerical assessment method for wrinkles by image processing. Tsuji et al. [21] demonstrated the histological changes around a wrinkle due to aging by in vitro microscopy. Tsukahara et al. $[22,23]$ assessed facial wrinkles to identify the grades of cutaneous aging. Batisse et al. [24] addressed the age-related change in wrinkling capacity from histological and mechanical standpoints. However, only a 
small number of studies have explicitly addressed the wrinkle formation mechanism with aging.

Magnenat-Thalmann et al. [25] carried out a finite element analysis of skin wrinkling with a three-layer model and showed a gradual enlargement of the buckling length with aging. However, their analyses considered only one buckling mode, and a gradual enlargement of wrinkles is not consistent with our experience, which indicates that prominent wrinkles suddenly appear at a certain age.

For the assessment of wrinkle properties, we propose a new concept of multistage buckling with a five-layered skin model, which is necessary to evaluate the mechanical property of skin from the micromechanical standpoint. Moreover, we perform a sensitivity analysis that helps explain the effect of age-related changing of the skin structure on the wrinkle property. Through a numerical example of human facial skin, we discuss the reason why a wrinkle becomes prominent in aged skin.

\section{Methods}

\subsection{Structure of skin and five-layer model}

Human skin consists of three layers: epidermis, dermis and hypodermis. The epidermis is biologically divided into four layers: stratum corneum (horny layer), granular layer, prickle layer, and basal layer. The dermis consists of three layers: papillary, subpapillary, and reticular layers. The hypodermis is a subcutaneous fat layer.

The stratum corneum is a dead cell layer and is extremely stiffer than the other layers; therefore, it should be considered an independent layer. The difference in the mechanical properties of the other layers in the epidermis are not clear, so they are unified in the mechanical sense and called the viable epidermis. The papillary and subpapillary layers consist of thin collagens and rich elastic fibers, and hold a lot of water [6,8]; so, these layers are flexible relative to the reticular layer, which consists of thick collagen bundles connected to each other by large elastic fibers $[6,13]$. Consequently, skin can be divided into five mechanically distinct layers: the stratum corneum (SC), viable epidermis (VE), papillary dermis (PD), reticular dermis (RD), and hypodermis (HD). We assume that each layer is homogeneous and isotropic, while its stiffness and thickness alter with aging. Since our interest is not on accurate behavior of skin but on fundamental properties, the viscosity and nonlinearity are neglected. The effect of pretension is also neglected for simplicity.

\subsection{Multistage buckling theory}

We interpret a skin wrinkle as a consequence of the buckling of skin. A permanent wrinkle is, of course, affected by many factors such as remodeling, adaptation, and degradation with aging. In principle, however, a wrinkle is initiated by the repetition of buckling caused by a muscle contraction at the same site. Therefore, the buckling property is the most important factor for wrinkle formation.
Table 1

Layer division of bilayer model in three buckling stages.

\begin{tabular}{|c|c|c|c|c|}
\hline & Stage I & Stage II & Stage IIIA & Stage IIIB \\
\hline SC & Buckling & & \multirow{4}{*}{ Buckling } & Dixid \\
\hline $\mathrm{VE}$ & Foundation & BuCkIIng & & Kigia \\
\hline PD & \multirow{3}{*}{ Ineffective } & Foundation & & Foundation \\
\hline $\mathrm{RD}$ & & \multirow{2}{*}{ Ineffective } & & Buckling \\
\hline HD & & & Foundation & Foundation \\
\hline
\end{tabular}

SC: stratum corneum, VE: viable epidermis, PD: papillary dermis, RD: reticular dermis, HD: hypodermis

The upper layer of skin is stiffer and thinner than the lower layer in general. Therefore, for uniform compression, buckling occurs at the upper layer first. Subsequently, the lower layer experiences buckling against the larger compression. We define such distinct phases of buckling as multistage buckling with three stages. The interaction between stages is neglected. The categorization is summarized in Table 1 . Stage I is horny buckling, where SC buckles under the support of VE. Stage II is epidermal buckling, where the upper two layers buckle simultaneously under the support of PD. Stage III is dermal buckling, where the upper four layers buckle with HD as a foundation. Moreover, Stage III has another possibility that RD buckles under the support of $\mathrm{PD}$ and HD. The former is referred to as Stage IIIA, and the latter is Stage IIIB. The bottom of skin is supposed to be fixed to the muscle membrane.

$\mathrm{PD}$ is not considered the lowest layer in the buckled region, because $\mathrm{PD}$ is softer than the underlying $\mathrm{RD}$. To describe the buckling behavior in each stage, we employ a bilayer model. Only one layer under the buckled layers is assumed to be the elastic foundation, because the effective depth of the foundation is shallow by the effect of shear stiffness, that is, the Saint Venant's principle. In Stage I, PD may affect the horny buckling since it is softer than VE. However, including PD in the foundation may leads to much underestimation of the stiffness of foundation, because PD is very thick especially in aged facial skin. Therefore, we employ one-layer foundation. The shear stiffness of foundation is also considered.

To find a specific property of skin, we assume infinitely expanding flat skin that is subjected to a uniaxial compression induced geometrically by a muscle contraction. Since the compression is uniaxial, the problem can be reduced to two-dimensional one, which is described as a compressed beam on a two-parameter foundation [26,27], as shown in Fig. 1. Deformation of the beam infinitely continues to the width direction, and we employ a unit width in calculation. The elastic foundation consists of continuously distributed linear

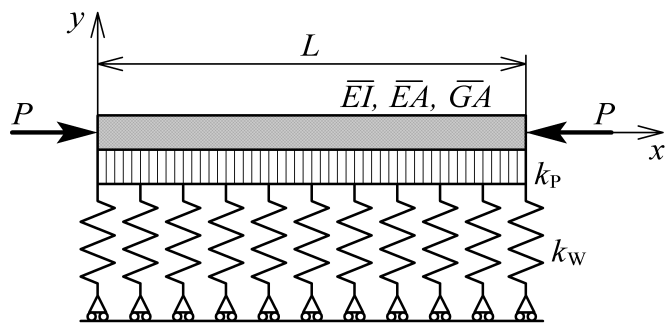

Fig. 1. Bilayer buckling model represented by a Timoshenko beam on a two-parameter elastic foundation. A series of vertical springs expresses the continuous foundation. The intermediate layer is a layer to express the shear stiffness of the foundation. The bottom of foundation is vertically fixed on the muscle membrane. 
springs and shear layer to represent shear interaction between springs. Horizontal compression of the foundation is neglected.

Assuming that each layer is linearly elastic and the deformation before buckling is small enough, we carry out a linear buckling analysis. Since skin is very flexible and shearing deformation of beam is not negligible, we employ Timoshenko beam. Internal compressive force in the beam is denoted by $P$. The flexural rigidity and shear stiffness of the composite beam is denoted by $\overline{E I}$ and $\overline{G A}$, respectively. The spring constant, namely Winkler constant, is denoted by $k_{\mathrm{W}}$, and the shear constant of foundation, namely Pasternak constant, is denoted by $k_{\mathrm{P}}$. These parameters depend on the buckling stage. The beam length is denoted by $L$, which is infinitely large in this problem.

Denoting the deflection of the beam by $v$, one can find the buckling equation as

$$
\left(1-\frac{P-k_{\mathrm{p}}}{\kappa \overline{G A}}\right) \frac{\mathrm{d}^{4} v}{\mathrm{~d} x^{4}}+\left(\frac{P-k_{\mathrm{p}}}{\overline{E I}}-\frac{k_{\mathrm{W}}}{\kappa \overline{G A}}\right) \frac{\mathrm{d}^{2} v}{\mathrm{~d} x^{2}}+\frac{k_{\mathrm{W}}}{\overline{E I}} v=0
$$

where $\kappa$ is the shear correction factor. In this study, $\kappa=0.854$ is used for a rectangular cross-section [28]. The minimum buckling load for a periodic solution [29] is obtained by

$$
P_{\mathrm{cr}}=k_{\mathrm{P}}+\left(2-\frac{\sqrt{k_{\mathrm{w}} \overline{\overline{E I}}}}{\kappa \overline{G A}}\right) \sqrt{k_{\mathrm{W}} \overline{\overline{E I}}} .
$$

This is realized when the beam length satisfies

$$
L=\alpha L_{\text {cr }}
$$

where $\alpha$ is a positive integer, and $L_{\mathrm{cr}}$ is the wavelength of the minimum-buckling mode, which is obtained by

$$
L_{\mathrm{cr}}=2 \pi \sqrt{1-\frac{\sqrt{k_{\mathrm{W}} \overline{\overline{E I}}}}{\kappa \overline{G A}}} \sqrt[4]{\frac{\overline{E I}}{k_{\mathrm{W}}}} .
$$

The infinite-length beam corresponds to the case that $\alpha$ is infinitely large. The other buckling modes have higher buckling loads than $P_{\mathrm{cr}}$ in Eq. (2), even if the wavelength of the buckling mode is longer than $L_{\mathrm{cr}}$. Thus, $L_{\mathrm{cr}}$ is the characteristic buckling length that most easily appears for the infinite-length skin. We hereafter refer to $L_{\mathrm{cr}}$ as the specific wrinkle size.

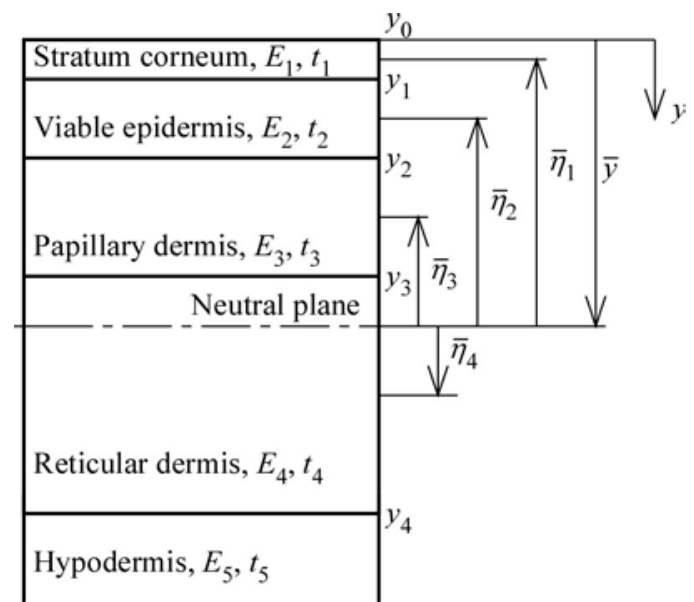

Fig. 2. Coordinates of five-layered skin model for Stage III. $y_{i}$ denotes the position of the interface relative to the top surface. $\bar{y}$ is the position of the neutral plane for the beam layer, and $\bar{\eta}_{i}$ is the distance from the neutral plane to the center of the $i$-th layer.
The critical load is not a comparable parameter between buckling stages because each stage has an individual beam model dependent on the buckled layers. For a comparable parameter, instead, we adopt the critical strain obtained by

$$
\varepsilon_{\text {cr }}=\frac{k_{\mathrm{P}}}{\overline{E A}}+\left(2-\frac{\sqrt{k_{\mathrm{w}} \overline{\overline{E I}}}}{\kappa \overline{G A}}\right) \frac{\sqrt{k_{\mathrm{W}} \overline{\overline{E I}}}}{\overline{E A}}
$$

where $\overline{E A}$ is the tensile stiffness of the composite beam. The buckling that has the smallest critical strain in the three stages most often appears on the skin and strongly affects the formation of permanent wrinkles of that stage.

Five material parameters $\overline{E I}, \overline{E A}, \overline{G A}, k_{\mathrm{W}}$, and $k_{\mathrm{P}}$ are obtained as follows. The Young's modulus, shear modulus and thickness of the $i$-th upper layer are denoted by $E_{i} G_{i}$ and $t_{i}$, respectively. The coordinate system and geometrical configuration are illustrated in Fig. 2. The suffixes in Fig. 2 are for Stage IIIA. The origin of the $y$-axis is placed at the top surface, and the $i$-th interface position is denoted by $y_{i}$, where $y_{0}=0$. The position of the neutral plane of the beam is denoted by $\bar{y}$, which is obtained by

$$
\bar{y}=\frac{1}{2 \overline{E A}} \sum_{i=a}^{n} E_{i}\left(y_{i}^{2}-y_{i-1}^{2}\right) .
$$

where $a$ and $n$ are the uppermost layer number and the bottom layer number in the beam. The flexural rigidity is obtained by the sum of the flexural rigidity of each layer around the neutral axis:

$$
\overline{E I}=\sum_{i=a}^{n} E_{i}\left(\frac{t_{i}^{3}}{12}+\bar{\eta}_{i}^{2} t_{i}\right)
$$

where $\bar{\eta}_{i}$ is the distance from the neutral plane to the central plane of the $i$-th layer. Since the compressive strain is assumed to be uniform, the tensile stiffness is obtained by the Voigt approximation [30]:

$$
\overline{E A}=\sum_{i=a}^{n} E_{i} t_{i} \cdot
$$

Moreover, using the Reuss approximation [30], the shear stiffness of the beam is obtained by

$$
\frac{1}{\overline{G A}}=\left(\sum_{i=a}^{n} t_{i}\right)^{-2} \sum_{i=a}^{n} \frac{t_{i}}{G_{i}}
$$

The Winkler constant of the elastic foundation is simply determined by

$$
k_{\mathrm{W}}=\frac{E_{n+1}}{t_{n+1}} .
$$

Assuming the shear strain in the foundation linearly decreases in the depth direction and vanishes at the bottom of foundation, one obtains the Pasternak constant as

$$
k_{\mathrm{p}}=\frac{G_{n+1} t_{n+1}}{2} .
$$

Two foundation constants in Stage IIIB are obtained by simply adding the stiffness of PD and HD in the same manner.

\subsection{Sensitivity analysis}

To investigate the contribution of the change in 
Table 2

Mechanical parameters of five-layer model determined to be consisten with experimental results for human skin [16,25,32].

\begin{tabular}{lllll}
\hline \multirow{2}{*}{ Layer } & Young & \multicolumn{3}{c}{ Aged } \\
\cline { 2 - 5 } & $E_{i}[\mathrm{MPa}]$ & $t_{i}[\mathrm{~mm}]$ & $E_{i}[\mathrm{MPa}]$ & $t_{i}[\mathrm{~mm}]$ \\
\hline Stratum corneum & 6.000 & 0.02 & 12.00 & 0.02 \\
Viable epidermis & 0.136 & 0.18 & 0.272 & 0.08 \\
Papillary dermis & 0.040 & 0.20 & 0.040 & 0.50 \\
Reticular dermis & 0.080 & 1.10 & 0.160 & 1.10 \\
Hypodermis & 0.034 & 2.00 & 0.034 & 2.00 \\
\hline
\end{tabular}

mechanical parameters to the change in wrinkle properties, we carry out a sensitivity analysis of the specific wrinkle size and the critical strain with respect to Young's modulus and the thickness of each layer. The mechanical parameters alter with aging and their alteration is qualitatively known. Therefore, the sensitivity analysis quantifies the influence of such alteration on the wrinkle properties and may clarify the wrinkle formation mechanism. The derivatives are obtained straightforward by the direct differentiation. Therefore, the details of formulation are omitted.

\subsection{Aging effects and parameter setting}

We prepare two cases, one for young skin and one for aged skin, whose mechanical parameters are listed in Table 2. Cutaneous aging is categorized into two types: intrinsic aging and photoaging [11]. Intrinsic aging is chronological and genetically determined, and photoaging is a histological change caused by sun exposure. Because facial skin is exposed to sunlight, we consider both aging processes. The mechanical parameters in Table 2 were determined as described below.

Young's moduli for VE, RD and HD in young skin are set equal to the experimental values for the epidermis, dermis and hypodermis of finger pad skin [16]. Young's modulus of SC in both young and aged skins is set to the value used by Magnenat-Thalmann et al. [25]. We suppose that Young's moduli of SC, VE and RD double from young skin to aged skin [31], because intrinsic aging induces the loss of moisture and stiffening of the fibrous materials. Degradation of the keratinocytes is represented by a decrease in thickness of VE.

Takema et al. [32] obtained the statistical variation in the averaged thickness of facial skin with aging, as shown in Fig. 3. This thickness is the total for the

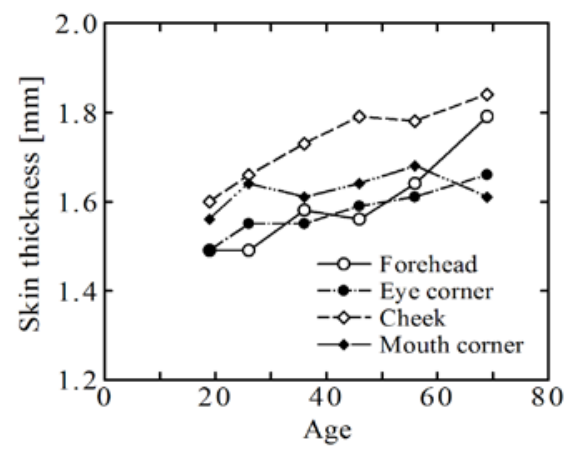

Fig. 3. Statistical variation of averaged total thickness of skin including the dermis and epidermis [32]. The thickness was measured at four facial sites: forehead, eye corner, cheek, and mouth corner by ultrasonography. epidermis and dermis. From this result, we determined the total thickness of the epidermis and dermis is equal to $1.5 \mathrm{~mm}$ for young skin and $1.7 \mathrm{~mm}$ for aged skin; these values are most similar to the eye corner. The total thickness is divided into each layer as mentioned below. The thickness of HD is strongly dependent on each individual, so we tentatively determined it as $2.0 \mathrm{~mm}$ for both young and aged skin.

As seen in Fig. 3, the thickness of facial skin increases with intrinsic aging and photoaging, while the thickness of sun-protected skin decreases with aging [32]. Hence, the cause of this thickening can be regarded as photoaging. Photoaging mainly leads to solar elastosis, which is an excessive generation of abnormal elastic fibers in PD [6,11,21,33]. This elastotic deterioration can also be detected as a SLEB (subepidermal low echogenic band) by ultrasonography [34-37]. The SLEB thickness increases with photoaging [37], so we assume that the thickening of facial skin is associated with the thickening of PD, while the thickness of RD is constant. Moreover, since PD is flexible, we assume that Young's modulus of $\mathrm{PD}$ is half of RD.

Summarizing the above, intrinsic aging is represented by the stiffening of SC, VE and $\mathrm{RD}$, and the thinning of VE. Photoaging is represented by the thickening of PD. Supposing that the young and aged skins in Table 2 correspond to 20 and 70 years old, respectively, these alterations are linearly interpolated with respect to the age. The chronological alteration is simulated to demonstrate the aging effect on the buckling property.

\section{Results}

The calculated $L_{\mathrm{cr}}$ and $\varepsilon_{\mathrm{cr}}$ for every stage of young and aged skins are summarized in Table 3. The $\overline{E I}$, $\overline{E A}, \overline{G A}, k_{\mathrm{W}}$ and $k_{\mathrm{P}}$ are also listed. Figures 4 and 5 show the chronological alteration of $L_{\mathrm{cr}}$ and $\varepsilon_{\mathrm{cr}}$, respectively. The Roman numeral in the superfix indicates the buckling stage like $L_{\mathrm{cr}}^{\mathrm{I}}$ and $L_{\mathrm{cr}}^{\mathrm{II}}$, hereafter. As the buckling stage progresses, $L_{\mathrm{cr}}$ increases because of a drastic increase in $\overline{E I}$. However, $L_{\mathrm{cr}}^{\mathrm{IIIA}}$ and $L_{\mathrm{cr}}^{\mathrm{IIIB}}$ vanished at a middle age, because $L_{\mathrm{cr}}^{\mathrm{IIIA}}$ in aged region and $L_{\mathrm{cr}}^{\mathrm{IIIB}}$ in young region became imaginary. This means there is no minimum buckling load, and the

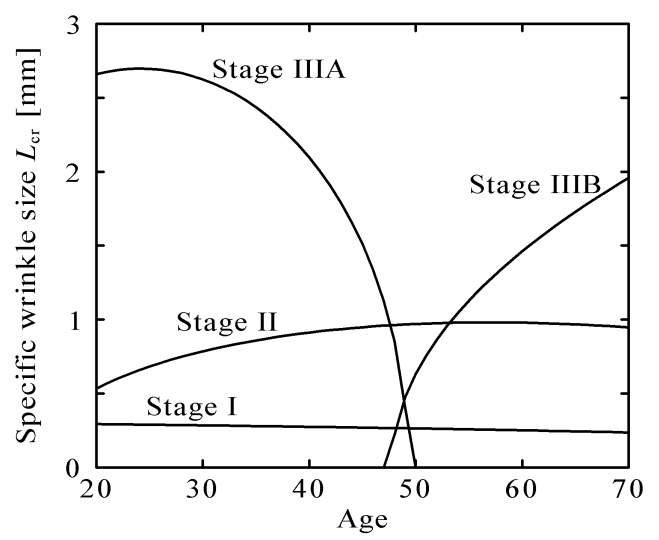

Fig. 4. Chronological alteration of the specific wrinkle sizes with aging in all Stages. 
Table 3

Calculated wrinkle properties of young and aged skins.

\begin{tabular}{|c|c|c|c|c|c|c|c|}
\hline \multicolumn{8}{|c|}{ Young skin (20 years old) } \\
\hline Stage & $L_{\mathrm{cr}}[\mathrm{mm}]$ & $\varepsilon_{\mathrm{Cr}}[\%]$ & $\overline{E I} \quad\left[\mathrm{Nmm}^{2}\right]$ & $\overline{E A} \quad[\mathrm{~N}]$ & $\overline{G A}[\mathrm{~N}]$ & $k_{\mathrm{W}}\left[\mathrm{N} / \mathrm{mm}^{2}\right]$ & $k_{\mathrm{P}}[\mathrm{N}]$ \\
\hline I & 0.294 & 6.47 & $4.00 \times 10^{-6}$ & $1.20 \times 10^{-1}$ & $4.29 \times 10^{-2}$ & $7.56 \times 10^{-1}$ & $4.37 \times 10^{-3}$ \\
\hline II & 0.535 & 7.11 & $2.73 \times 10^{-4}$ & $1.44 \times 10^{-1}$ & $1.08 \times 10^{-2}$ & $2.00 \times 10^{-1}$ & $1.43 \times 10^{-3}$ \\
\hline IIIA & 2.659 & 19.12 & $5.58 \times 10^{-2}$ & $2.40 \times 10^{-1}$ & $4.00 \times 10^{-2}$ & $1.70 \times 10^{-2}$ & $1.21 \times 10^{-2}$ \\
\hline IIIB & Imaginary & (33.63) & $8.87 \times 10^{-3}$ & $8.80 \times 10^{-2}$ & $3.14 \times 10^{-2}$ & $2.17 \times 10^{-1}$ & $1.36 \times 10^{-2}$ \\
\hline \multicolumn{8}{|c|}{ Aged skin (70 years old) } \\
\hline Stage & $L_{\mathrm{cr}}[\mathrm{mm}]$ & $\varepsilon_{\mathrm{cr}}[\%]$ & $\overline{\overline{E I}}\left[\begin{array}{l}\left.\mathrm{Nmm}^{2}\right] \\
\end{array}\right.$ & $\overline{\overline{E A}}[\mathrm{~N}]$ & $\overline{\overline{G A}}[\mathrm{~N}]$ & $k_{\mathrm{W}}\left[\mathrm{N} / \mathrm{mm}^{2}\right]$ & $k_{\mathrm{P}}[\mathrm{N}]$ \\
\hline I & 0.237 & 5.81 & $8.00 \times 10^{-6}$ & $2.40 \times 10^{-1}$ & $8.57 \times 10^{-2}$ & 3.40 & $3.89 \times 10^{-3}$ \\
\hline II & 0.947 & 2.96 & $6.95 \times 10^{-5}$ & $2.62 \times 10^{-1}$ & $1.21 \times 10^{-2}$ & $8.00 \times 10^{-2}$ & $3.57 \times 10^{-3}$ \\
\hline IIIA & Imaginary & $(12.24)$ & $1.54 \times 10^{-1}$ & $4.58 \times 10^{-1}$ & $5.25 \times 10^{-2}$ & $1.70 \times 10^{-2}$ & $1.21 \times 10^{-2}$ \\
\hline IIIB & 1.958 & 37.86 & $1.77 \times 10^{-2}$ & $1.76 \times 10^{-1}$ & $6.29 \times 10^{-2}$ & $9.70 \times 10^{-2}$ & $1.57 \times 10^{-2}$ \\
\hline
\end{tabular}

Table 4

Sensitivities with respect to Young's modulus of each layer (the value in parentheses is for aged skin).

\begin{tabular}{|c|c|c|c|c|c|c|c|c|}
\hline & $\partial L_{\mathrm{cr}}^{\mathrm{I}} / \partial E_{i}$ & {$[\mathrm{~mm} / \mathrm{MPa}]$} & $\partial L_{\mathrm{cr}}^{\mathrm{II}} / \partial E_{i}$ & {$[\mathrm{~mm} / \mathrm{MPa}]$} & $\partial L_{\mathrm{cr}}^{\mathrm{IIIA}} / \partial E_{i}$ & {$[\mathrm{~mm} / \mathrm{MPa}]$} & $\partial L_{\mathrm{cr}}^{\mathrm{IIIB}} / \partial E_{i}$ & {$[\mathrm{~mm} / \mathrm{MPa}]$} \\
\hline Stratum corneum: $E_{1}$ & $1.29 \times 10^{-2}$ & $\left(5.32 \times 10^{-3}\right)$ & $-9.28 \times 10^{-3}$ & $\left(2.49 \times 10^{-3}\right)$ & $-2.56 \times 10^{-1}$ & $(-)$ & & \\
\hline Viable epidermis: $E_{2}$ & $-5.68 \times 10^{-1}$ & $\left(-2.35 \times 10^{-1}\right)$ & 5.43 & $(1.02)$ & 4.62 & $(-)$ & & \\
\hline Papillary dermis: $E_{3}$ & & & -17.1 & $(-7.68)$ & 75.3 & $(-)$ & - & $(-44.5)$ \\
\hline Reticular dermis: $E_{4}$ & & & & & 57.8 & $(-)$ & - & (13.5) \\
\hline \multirow[t]{2}{*}{ Hypodermis: $E_{5}$} & & & & & -198 & $(-)$ & - & $(-11.1)$ \\
\hline & $\partial \varepsilon_{\mathrm{cr}}^{\mathrm{I}} / \partial E_{i}$ & {$[\% / \mathrm{MPa}]$} & $\partial \varepsilon_{\mathrm{cr}}^{\mathrm{II}} / \partial E_{i}$ & [\%/MPa] & $\partial \varepsilon_{\mathrm{cr}}^{\mathrm{IIIA}} / \partial E_{i}$ & {$[\% / \mathrm{MPa}]$} & $\partial \varepsilon_{\mathrm{cr}}^{\mathrm{IIIB}} / \partial E_{i}$ & {$[\% / \mathrm{MPa}]$} \\
\hline Stratum corneum $E_{1}$ & $-8.37 \times 10^{-1}$ & $\left(-3.03 \times 10^{-1}\right)$ & $-9.59 \times 10^{-1}$ & $\left(-2.16 \times 10^{-1}\right)$ & -1.53 & $(-)$ & & \\
\hline Viable epidermis $E_{2}$ & 36.9 & (13.4) & 27.7 & (1.96) & -8.42 & $(-)$ & & \\
\hline Papillary dermis $E_{3}$ & & & 49.8 & (51.5) & 56.0 & $(-)$ & - & $(161)$ \\
\hline Reticular dermis $E_{4}$ & & & & & 22.1 & $(-)$ & - & $(-89.3)$ \\
\hline Hypodermis $E_{5}$ & & & & & 186 & $(-)$ & - & $(231)$ \\
\hline
\end{tabular}

Table 5

Sensitivities with respect to the thickness of each layer (the value in parentheses is for aged skin).

\begin{tabular}{|c|c|c|c|c|c|c|c|c|}
\hline & $\partial L_{\mathrm{cr}}^{\mathrm{I}} / \partial t_{i}$ & {$[\mathrm{~mm} / \mathrm{mm}]$} & $\partial L_{\mathrm{cr}}^{\mathrm{II}} / \partial t_{i}$ & {$[\mathrm{~mm} / \mathrm{mm}]$} & $\partial L_{\mathrm{cr}}^{\mathrm{IIIA}} / \partial t_{i}$ & {$[\mathrm{~mm} / \mathrm{mm}]$} & $\partial L_{\mathrm{cr}}^{\mathrm{IIIB}} / \partial t_{i}$ & {$[\mathrm{~mm} / \mathrm{mm}]$} \\
\hline Stratum corneum: $t_{1}$ & 10.8 & (8.67) & 4.23 & (8.54) & -65.0 & $(-)$ & & \\
\hline Viable epidermis: $t_{2}$ & $4.29 \times 10^{-1}$ & $\left(7.98 \times 10^{-1}\right)$ & -1.29 & (5.87) & 1.64 & $(-)$ & & \\
\hline Papillary dermis: $t_{3}$ & & & 3.41 & $\left(6.14 \times 10^{-1}\right)$ & -8.74 & $(-)$ & - & (3.56) \\
\hline Reticular dermis: $t_{4}$ & & & & & -1.19 & $(-)$ & - & $\left(-1.80 \times 10^{-1}\right)$ \\
\hline \multirow[t]{2}{*}{ Hypodermis: $t_{5}$} & & & & & 3.36 & $(-)$ & - & $\left(1.89 \times 10^{-1}\right)$ \\
\hline & $\partial \varepsilon_{\mathrm{cr}}^{\mathrm{I}} / \partial t_{i}$ & {$[\% / \mathrm{mm}]$} & $\partial \varepsilon_{\mathrm{cr}}^{\mathrm{II}} / \partial t_{i}$ & {$[\% / \mathrm{mm}]$} & $\partial \varepsilon_{\mathrm{cr}}^{\mathrm{IIIA}} / \partial t_{i}$ & {$[\% / \mathrm{mm}]$} & $\partial \varepsilon_{\mathrm{cr}}^{\mathrm{IIIB}} / \partial t_{i}$ & {$[\% / \mathrm{mm}]$} \\
\hline Stratum corneum $t_{1}$ & -113 & $(20.0)$ & -239 & $(-108)$ & -443 & $(-)$ & & \\
\hline Viable epidermis $t_{2}$ & 12.6 & $(-4.99)$ & 26.6 & (18.5) & 2.71 & $(-)$ & & \\
\hline Papillary dermis $t_{3}$ & & & $-6.55 \times 10^{-2}$ & (1.34) & $1.36 \times 10^{-1}$ & $(-)$ & - & $(-4.77)$ \\
\hline Reticular dermis $t_{4}$ & & & & & 4.14 & $(-)$ & - & $(-3.25)$ \\
\hline Hypodermis $t_{5}$ & & & & & 1.89 & $(-)$ & - & $(2.98)$ \\
\hline
\end{tabular}

buckling mode is not periodic. This phenomenon was caused by small $\overline{G A}$ relative to $\overline{E I}$ or $k_{\mathrm{w}}$, that is, a shear instability. However, note that the evaluation of accurate behavior in Stage III needs to consider the nonlinearity and interaction between different stages, since the compression is very large.

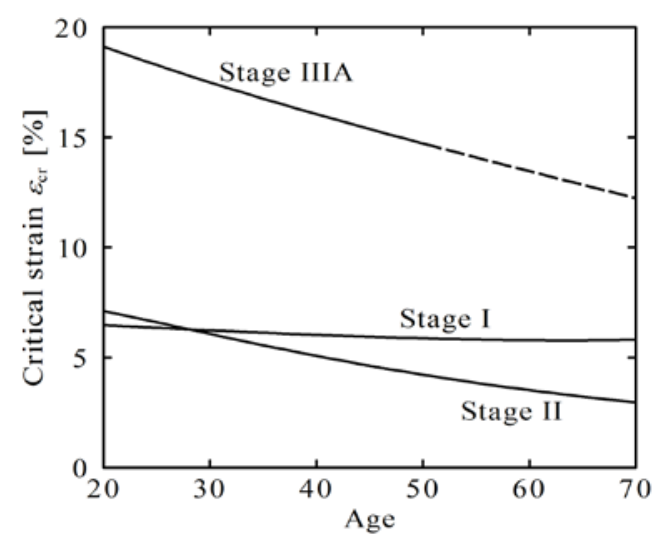

Fig. 5. Chronological alteration of the critical strains with aging in all Stages other than Stage IIIB. Broken line indicates a meaningless part because of the imaginary wavelength.
The $L_{\text {cr }}^{\mathrm{I}}$ is close to the spacing of furrow lines, which are so small as to be obscure and not recognized as a wrinkle. The $L_{\mathrm{cr}}^{\mathrm{II}}$ is also small but slightly prominent in aged skin such as so-called small wrinkle. The $L_{\mathrm{cr}}^{\mathrm{IIIA}}$ and $L_{\mathrm{cr}}^{\mathrm{IIIB}}$ are similar to the spacing of large wrinkles.

The $\varepsilon_{\mathrm{cr}}^{\mathrm{I}}$ was the smallest in young skin, while $\varepsilon_{\mathrm{cr}}^{\mathrm{II}}$ was the minimum in aged skin. That is to say, Stage I wrinkle most easily appears in young skin, whereas Stage II wrinkle most readily appears in aged skin. This means that the small wrinkle becomes dominant instead of the discreet furrow in aged skin. The mode switch from Stage I to Stage II is seen in Fig. 5 as a crossing point near 30 years old. Moreover, this result supports the fact that the small wrinkles, such as crow's-feet, suddenly appear with aging on the site of expressive wrinkle, where buckling is frequently repeated. This repetition of buckling enhances the opportunity to yield Stage II buckling in aged skin and to damage the epidermis and dermis more significantly than Stage I in young skin. Thus, the mode switch gives rise to the sudden 
appearance of permanent small wrinkles.

The calculated sensitivities are listed in Tables 4 and 5. The sensitivities for the aged skin are put in parentheses. In this example, $L_{\mathrm{cr}}$ and $\varepsilon_{\mathrm{cr}}$ were most sensitive to Young's modulus of foundation layer, and their sensitivities were negative for $L_{\mathrm{cr}}$ and positive for $\varepsilon_{\mathrm{cr}}$. Therefore, softening or thickening of foundation layer makes lager wrinkles appear more easily. Moreover, the thickness of SC has the highest sensitivity to $L_{\mathrm{cr}}$ and $\varepsilon_{\mathrm{cr}}$, because Young's modulus of SC is highest and mostly affects the stiffness of beam.

\section{Discussion}

Human skin tends to be stiffer with aging in general [32]. Therefore, typical skin has a latent capability to enlarge $L_{\mathrm{cr}}$ with aging as discussed by Magnenat-Thalmann et al. [25]. The wider wrinkle has the larger amplitude in post-buckling deformation, so it enables the epidermis to penetrate deeper into the dermis. Thus, a wide and deep wrinkle causes significant damages in the skin and yields a permanent wrinkle.

The most important finding in this study is that the possibility of mode switch from Stage I to Stage II was elucidated by using a five-layer model. Magnenat-Thalmann et al. [25] could not observe the mode switch, because they used a three-layer model. In Fig. 5, the curves of Stage I and Stage II are close, so the switching point is easily changes depending on the model parameters. For instance, considering the effect of PD in Stage I leads to decrease in $\varepsilon_{\mathrm{cr}}^{\mathrm{I}}$. Then, the switching point shifts older. In such sense, the numerical results are not reliable yet. But the idea of mode switch may be an important key to clarify the wrinkle formation mechanism.

From the result of sensitivity analysis, the thinning of VE decreases $\varepsilon_{\mathrm{cr}}^{\mathrm{II}}$ about doubly than $\varepsilon_{\mathrm{cr}}^{\mathrm{I}}$, and the stiffening of VE increases $\varepsilon_{\mathrm{cr}}^{\mathrm{I}}$ more largely than $\varepsilon_{\mathrm{cr}}^{\mathrm{II}}$. Moreover, the thickening of PD by photoaging influences on the decrease in $\varepsilon_{\mathrm{cr}}^{\mathrm{II}}$. The stiffening of SC also facilitates the mode switch.

The mode switch causes a sudden appearance of permanent small wrinkles, as mentioned earlier. From an aesthetic or cosmetic viewpoint, therefore, the reduction of $\varepsilon_{\mathrm{cr}}^{\mathrm{I}}$ and the enlargement of $\varepsilon_{\mathrm{cr}}^{\mathrm{II}}$ are preferable for anti-wrinkle. To prevent the mode switch, some skincare or therapy against the aging effects is desired to protect the epidermis from stiffening by dehydration and thinning by degradation of the turnover, and to protect the dermis from thickening by photodamage. These findings are already well known as empirical information, but they were mechanically proven for the first time in this study with a new concept of mode switch.

\section{Conflict of Interest Statement}

All authors state that there are none.

\section{References}

[1] Butler RN, Fossel M, Harman SM, Heward CB, Olshansky SJ,
Perls TT, Rothman DJ, Rothman SM, Warner HR, West MD, Wright WE. Is there an antiaging medicine?. Journal of Gerontology: Biological Sciences 2002; 57A: B333-B338.

[2] Grossman T. Latest advances in antiaging medicine. The Keio Journal of Medicine 2005; 54: 85-94.

[3] Klatz R. New horizons for the clinical specialty of anti-aging medicine, The future with biomedical technologies. Annals of the New York Academy of Sciences 2005; 1057: 536-544.

[4] Micans P. The need for anti-aging medicine, The challenges faced to incorporate preventative medicine into the clinic and into society. Annals of the New York Academy of Sciences 2005; 1057: 545-562.

[5] Baumann L. Cosmetic dermatology, principles and practice. New York: McGraw-Hill Medical Publishing Division; 2002.

[6] Miyachi Y, Matsunaga K, Furukawa F, Utsugi R, editors. Aesthetic dermatology. Tokyo: Nanzando; 2005.

[7] Tagami H, Miyachi Y, Takigawa M, editors. Skin-care NO JISSAI. Tokyo: Bunkodo; 1999.

[8] Yasuda T, Urushibata O. BIYO NO HIFUKAGAKU, 8th edition. Tokyo: Nanzando; 2002.

[9] Burgess CM, editor. Cosmetic dermatology. Berlin: Springer; 2005.

[10] Baran R, Maibach HI, editors. Textbook of cosmetic dermatology, 3rd edition. London: Taylor \& Francis; 2005.

[11] Kligman AM, Takase Y, editors. Cutaneous aging. Tokyo: University of Tokyo Press; 1988.

[12] Braverman IM, Fonferko E. Studies in cutaneous aging: I. The elastic fiber network. The Journal of Investigative Dermatology 1982; 78: 434-443.

[13] Agache P, Humbert P, editors. Measuring the Skin. Berlin: Springer; 2004.

[14] Diridollou S, Black D, Lagarde JM, Gall Y, Berson M, Vabre V, Patat F, Vaillant L. Sex- and site-dependent variations in the thickness and mechanical properties of human skin in vivo. International Journal of Cosmetic Science 2000; 22: 421-435.

[15] Diridollou S, Vabre V, Berson M, Vaillant L, Black D, Lagarde JM, Gregoire JM, Gall Y, Patat F. Skin ageing: Change of physical properties of human skin in vivo. International Journal of Cosmetic Science 2001; 23: 353-362.

[16] Maeno T, Kobayashi K, Yamazaki N. Relationship between structure of finger tissue and location of tactile receptors. Transactions of the Japan Society of Mechanical Engineers 1997; 63C: 881-888.

[17] Hendriks FM, Brokken D, van Eemeren JTWM, Oomens CWJ, Baaijens FPT, Horsten JBAM. A numerical-experimental method to characteraize the non-linear mechanical behavior of human skin. Skin Research and Technology 2003; 9: 274-283.

[18] Hendriks FM, Brokken D, Oomens CWJ, Baaijens FPT. Influence of hydration and experimental length scale on the mechanical response of human skin in vivo, using optical coherence tomography. Skin Research and Technology 2004; 10: 231-241.

[19] Hendriks FM, Brokken D, Oomens CWJ, Bader DL, Baaijens FPT. The relative contributions of different skin layers to the mechanical behavior of human skin in vivo using suction experiments. Medical Engineering \& Physics 2006; 28: 259-266.

[20] Lagarde JM, Rouvrais C, Black D. Topography and anisotropy of the skin surface with ageing. Skin Research and Technology 2005; 11: $110-119$.

[21] Tsuji T, Yorifuji T, Hayashi Y, Hamada T. Light and scanning electron microscopic studies on wrinkles in aged persons' skin. British Journal of Dermatology 1986; 114: 329-335.

[22] Tsukahara K, Takema Y, Fujimura T, Moriwaki S, Kitahara T, Imokawa G. Determination of age-related changes in the morphological structure (sagging) of the human cheek using a photonumeric scale and three-dimensional surface parameters. International Journal of Cosmetic Science 2000; 22: 247-258.

[23] Tsukahara K, Takema Y, Fujimura T, Moriwaki S, Hattori M. Quantitative two-dimensional analysis of facial wrinkles of Japanese women at various ages. International Journal of Cosmetic Science 2002; 24: 71-80.

[24] Batisse D, Bazin R, Baldeweck T, Querleux B, Lévêque JL. Influence of age on the wrinkling capacities of skin. Skin Research and Technology 2002; 8: 148-154.

[25] Magnenat-Thalmann N, Kalra P, Lévêque JL, Bazin R, Batisse D, Querleux B. A computational skin model: Fold and wrinkle formation. IEEE Transactions on Information Technology in Biomedicine 2002; 6: 317-323.

[26] Kerr AD. Elastic and viscoelastic foundation models. Journal of Applied Mechanics 1964; 31: 491-498. 
[27] Wang YH, Tham LG, Cheung YK. Beams and plates on elastic foundations: A review. Progress in Structural Engineering and Materials 2005; 7: 174-182.

[28] Cowper GR. The shear coefficient in Timoshenko's beam theory. Journal of Applied Mechanics 1966; 33: 335-340.

[29] Bazant ZP, Cedolin L. Stability of structures. New York: Dover; 2003.

[30] Aboudi J. Mechanics of composite materials. Amsterdam: Elsevier; 1991.

[31] Agache PG, Monneur C, Leveque JL, De Rigal J. Mechanical properties and Young's modulus of human skin in vivo. Archives of Dermatological Research 1980; 269: 221-232.

[32] Takema Y, Yorimoto Y, Kawai M, Imokawa G. Age-related changes in the elastic properties and thickness of human facial skin. British Journal of Dermatology 1994; 131: 641-648.

[33] Imokawa G, Takema Y, Yorimoto Y, Tsukahara K, Kawai M, Imayama S. Degree of ultraviolet-induced tortuosity of elastic fibers in rat skin is age dependent. The Journal of Investigative Dermatology 1995; 105: 254-258.

[34] de Rigal J, Escoffier C, Querleux B, Faivre B, Agache P, Lévêque JL. Assessment of aging of the human skin by in vivo ultrasonic imaging. The Journal of Investigative Dermatrogy 1989; 93: 621-625.

[35] Gniadecka M, Gniadecki R, Serup J, Søndergaard J. Ultrasound structure and digital image analysis of the subepidermal low echogenic band in aged human skin: Diurnal changes and interindividual variability. The Journal of Investigative Dermatology 1994; 102: 362-365.

[36] Gniadecka M, Jemec GBE. Quantitative evaluation of chronological ageing and photoaging in vivo: Studies on skin echogenicity and thickness. British Journal of Dermatology 1998; 139: 815-821.

[37] Gniadecka M. Effects of ageing on dermal echogenicity. Skin Research and Technology 2001; 7: 204-207. 